\title{
Resolving the Physical Origin of Octahedral Tilting in Halide Perovskites
}

\author{
Jung-Hoon Lee, ${ }^{\dagger}$ Nicholas C. Bristowe, ${ }^{\ddagger}$ June Ho Lee, ${ }^{\S}$ Sung-Hoon Lee, ${ }^{\|}$Paul D. Bristowe, ${ }^{*}{ }^{\dagger}$ \\ Anthony K. Cheetham, ${ }^{* \dagger}$ and Hyun Myung Jang ${ }^{*, \S}$ \\ ${ }^{\dagger}$ Department of Materials Science and Metallurgy, University of Cambridge, Cambridge CB3 OFS, United Kingdom \\ ${ }^{\ddagger}$ Department of Materials, Imperial College London, London SW7 2AZ, United Kingdom \\ ${ }^{\S}$ Department of Materials Science and Engineering, and Division of Advanced Materials Science, Pohang University of Science and \\ Technology (POSTECH), Pohang 790-784, Republic of Korea \\ "Center for Artificial Low Dimensional Electronic Systems, Institute for Basic Science, Pohang 790-784, Republic of Korea
}

Supporting Information

ABSTRACT: Hybrid perovskites are currently the fastest growing photovoltaic technology, having reached a solar cell efficiency of over $20 \%$. One possible strategy to further improve the efficiency of perovskite solar cells is to tune the degree of octahedral tilting of the halide frame, since this in turn affects the optical band gap and carrier effective masses. It is commonly accepted that the ion sizes are the main control parameter influencing the degree of tilting in perovskites. Here we re-examine the origin of octahedral tilts in halide perovskites from systematic first-principles calculations. We find that while steric effects dominate the tilt magnitude in inorganic halides, hydrogen bonding between an organic A-cation and the halide frame plays a significant role in hybrids. For example, in the case of $\mathrm{MAPbI}_{3}$, our calculations suggest that, without the contribution from hydrogen bonding, the octahedra would not tilt at all. These results demonstrate that tuning the degree of hydrogen bonding can be used as an additional control parameter to optimize the photovoltaic properties of perovskites.

\section{INTRODUCTION}

Over the past decade, hybrid organic-inorganic perovskites such as methylammonium lead iodide $\left(\mathrm{MAPbI}_{3}, \mathrm{MA}=\right.$ $\mathrm{CH}_{3} \mathrm{NH}_{3}$ ) have received enormous interest in the scientific community as low cost and highly efficient solar cell materials. $^{1-11}$ To further advance their already impressive power conversion efficiency, ${ }^{12}$ it would be highly desirable to rationalize new design concepts by furthering our fundamental understanding of these materials. Through first-principles calculations and modeling the search for modified perovskites with optimized device properties can be streamlined. For example, it was predicted and confirmed experimentally ${ }^{13}$ that an intimate structure-property relationship exists in halide perovskites, whereby a certain cooperative structural distortion to the perovskite can systematically tune the electronic band structure including the band gap and band edge effective masses. ${ }^{13,14}$ By controlling the magnitude of this distortion, one can optimize various photovoltaic properties such as light absorption and electron-hole separation.

$\mathrm{MAPbI}_{3}$ undergoes several structural phase transitions with temperature, ${ }^{15}$ whereby the aforementioned cooperative distortions which govern the electronic properties, known as octahedral tilts, enter into the ground state. The octahedral tilts consist of a rigid rotation of the anion cage, and can appear around any of the three Cartesian directions in the crystal with

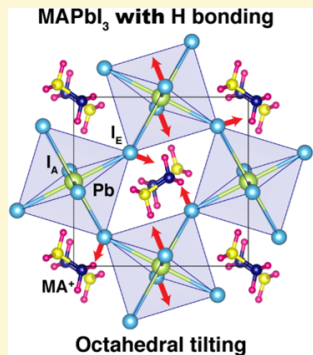

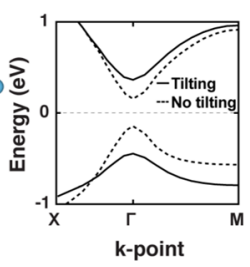

Schematic bands

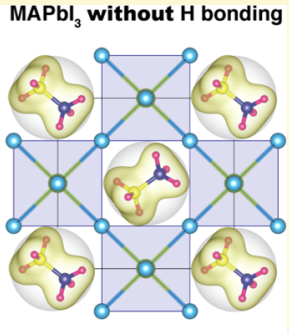

No octahedral tilting pubs.acs.org/cm 
a

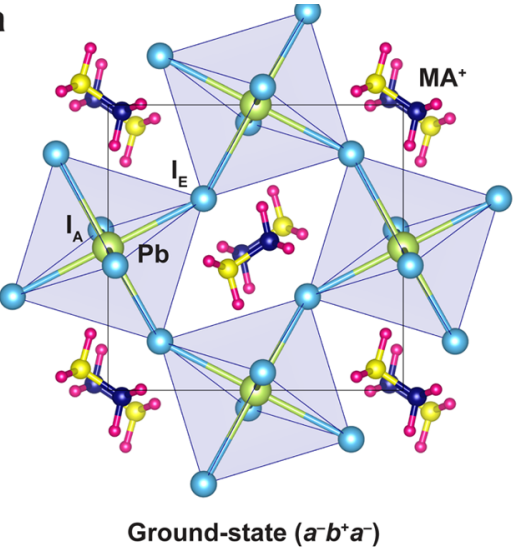

b

$\mathrm{Pbl}_{6}$

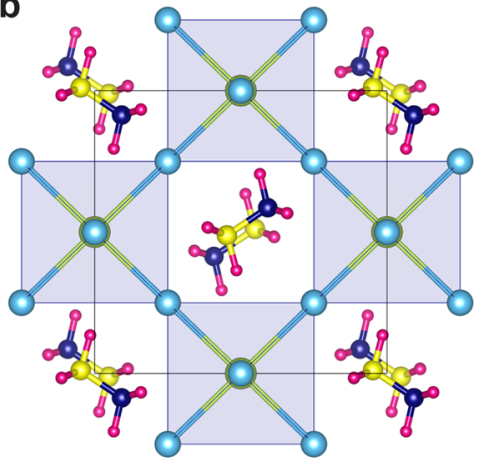

High-symmetry $\left(a^{0} a^{0} a^{0}\right)$

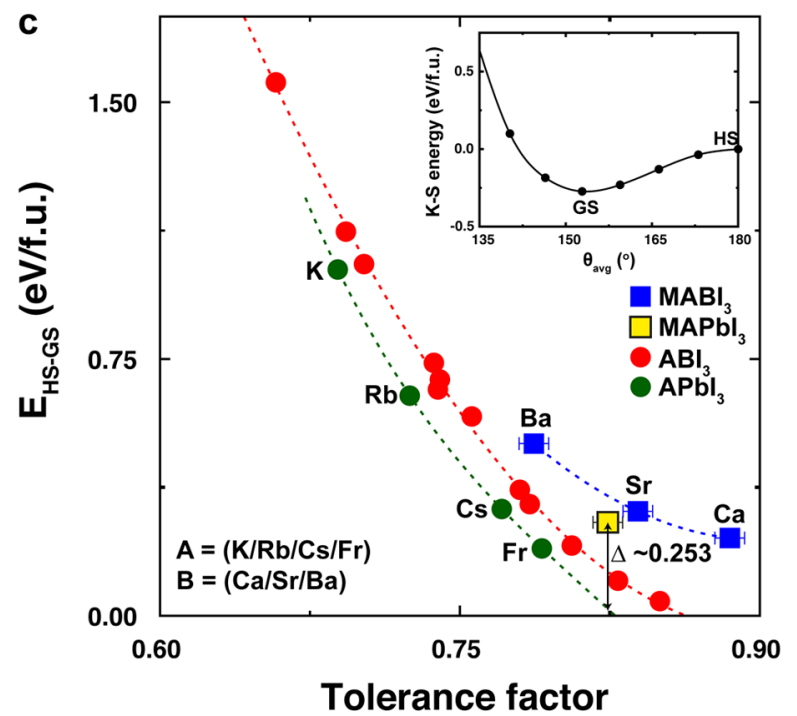

Figure 1. Effect of chemical substitution on the propensity for octahedral tilting in halide perovskites. (a) The ground state (GS) and (b) high symmetry (HS) structures of orthorhombic (o-) $\mathrm{MAPbI}_{3}$, composed of $\mathrm{PbI}_{6}$ octahedra and $\mathrm{MA}^{+}$groups. (c) The energy difference between the HS and the GS structures $\left(E_{\mathrm{HS}-\mathrm{GS}}\right)$ as a function of the tolerance factor for the $\mathrm{o}-\mathrm{A}(=\mathrm{K} / \mathrm{Rb} / \mathrm{Cs} / \mathrm{Fr}) \mathrm{B}(=\mathrm{Ca} / \mathrm{Sr} / \mathrm{Ba}) \mathrm{I}_{3}$ inorganic series $($ red circles $)$, o$\mathrm{APbI}_{3}$ inorganic series (green circles), o-MABI ${ }_{3}$ hybrid series (blue squares), and o-MAPbI ${ }_{3}$ (yellow square). The horizontal error bars of the o$\mathrm{MABI}_{3}$ hybrid structures represent the difference in tolerance factor calculated using the sphere and cylinder methods (see Methods section and SI Figure S1). Inset shows the computed Kohn-Sham $(\mathrm{K}-\mathrm{S})$ energies plotted as a function of the average tilt angle $\left(\theta_{\text {avg }}\right)$ between the equatorial $(\mathrm{Pb}-$ $\left.\mathrm{I}_{\mathrm{E}}-\mathrm{Pb}\right)$ and apical $\left(\mathrm{Pb}-\mathrm{I}_{\mathrm{A}}-\mathrm{Pb}\right)$ tilt angles for o- $\mathrm{MAPbI}_{3}$. SI Table S3 lists the ground state values of $\theta_{\text {avg }}$ for all the perovskites studied together with the values of $E_{\mathrm{HS}-\mathrm{GS}}$. The variation of $\theta_{\text {avg }}$ with tolerance factor and $E_{\mathrm{HS}-\mathrm{GS}}$ is given in SI Figure S2.

within this phase the MA ions are ordered, unlike the higher temperature tetragonal and cubic phases. However, our results are also relevant to tetragonal and cubic $\mathrm{MAPbI}_{3}{ }^{28}$ due to the dynamic appearance of these tilts as soft phonon modes. ${ }^{29} \mathrm{We}$ note that, for o- $\mathrm{MAPbI}_{3}$, neutron diffraction data ${ }^{30}$ confirm the ordered positions of the MA ions, and these agree well with our DFT calculations. We find a clear trend between A-cation size and the propensity to tilt in inorganic halides, in line with standard steric arguments, which we argue to be more accurately described as a second order Jahn-Teller effect whereby tilts aid orbital hybridizations. $\mathrm{Pb}$ lone pair bonding influences the strength of this steric (or more accurately second order Jahn-Teller) effect in lead-based halide perovskites. However, MA-I hydrogen bonding is shown to play an even more significant role in hybrid organic-inorganic lead halide perovskites. ${ }^{31}$ In the case of $\mathrm{MAPbI}_{3}$, our results suggest that without the contribution from $\mathrm{H}$-bonding the structure would remain untilted at all temperatures. We argue whether it is in fact coincidental that $\mathrm{MAPbI}_{3}$ shows an identical tilt pattern to many inorganic oxide perovskites, being driven instead by a particular pattern of highly directional hydrogen bonds. The results suggest that hydrogen bonding can be used as an additional control parameter to optimize photovoltaic properties, and electronic properties more generally, in perovskites.

\section{METHODS}

First-Principles Calculations. All the DFT calculations employed the generalized gradient approximation (GGA) implemented with projector augmented-wave (PAW) $)^{32,33}$ pseudopotentials as supplied in the Vienna Ab Initio Simulation Package (VASP)..$^{34-37}$ During relaxation, corrections for the van der Waals dispersion interaction (optB86b-vdW) were included. ${ }^{38,39}$ The spin-orbit coupling interaction was only included for the band structure and DOS calculations. The following parameters were adopted: (i) a $4 \times 3 \times 4$ MonkhorstPack k-point mesh centered at $\Gamma$, (ii) a $500 \mathrm{eV}$ plane-wave cutoff energy, and (iii) the tetrahedron method with Blochl corrections for the Brillouin zone integrations. ${ }^{40}$ The number of valence electrons treated explicitly were as follows: 14 for $\mathrm{Pb}\left(5 \mathrm{~d}^{10} 6 \mathrm{~s}^{2} 6 \mathrm{p}^{2}\right), 8$ for Ca $\left(3 p^{6} 4 s^{2}\right), 8$ for Ba $\left(5 p^{6} 6 s^{2}\right), 8$ for Sr $\left(4 p^{6} 5 s^{2}\right), 7$ for I $\left(5 s^{2} 5 p^{5}\right), 9$ for Cs $\left(5 s^{2} 5 p^{6} 6 s^{1}\right), 9$ for $\mathrm{K}\left(3 s^{2} 3 p^{6} 4 s^{1}\right), 9$ for $\mathrm{Rb}\left(4 s^{2} 4 p^{6} 5 s^{1}\right), 9$ for $\mathrm{Fr}$ $\left(6 s^{2} 6 p^{6} 7 s^{1}\right), 4$ for $\mathrm{C}\left(2 s^{2} 2 p^{2}\right), 5$ for $\mathrm{N}\left(2 s^{2} 2 p^{3}\right)$, and 1 for $\mathrm{H}\left(1 s^{1}\right)$. All 

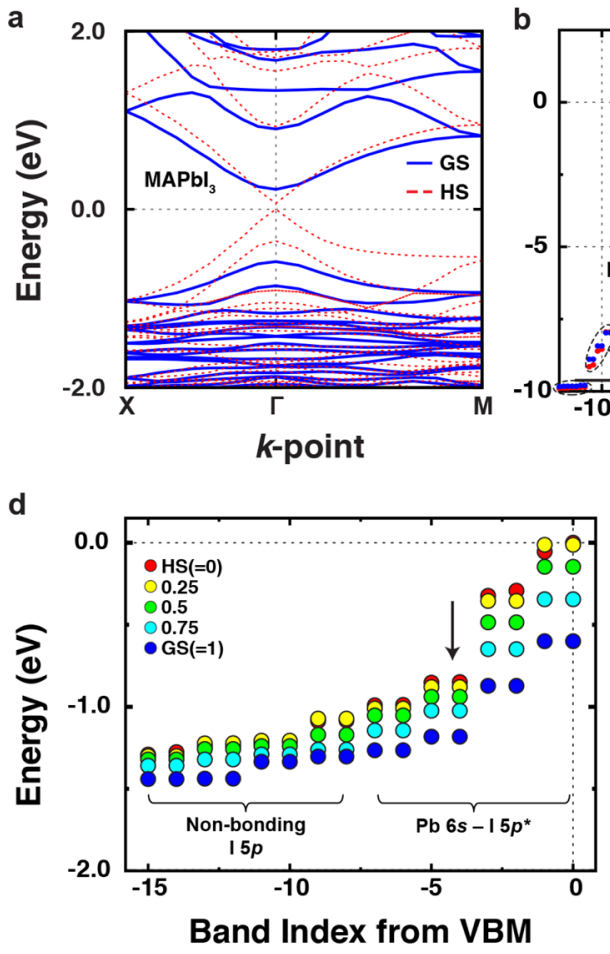
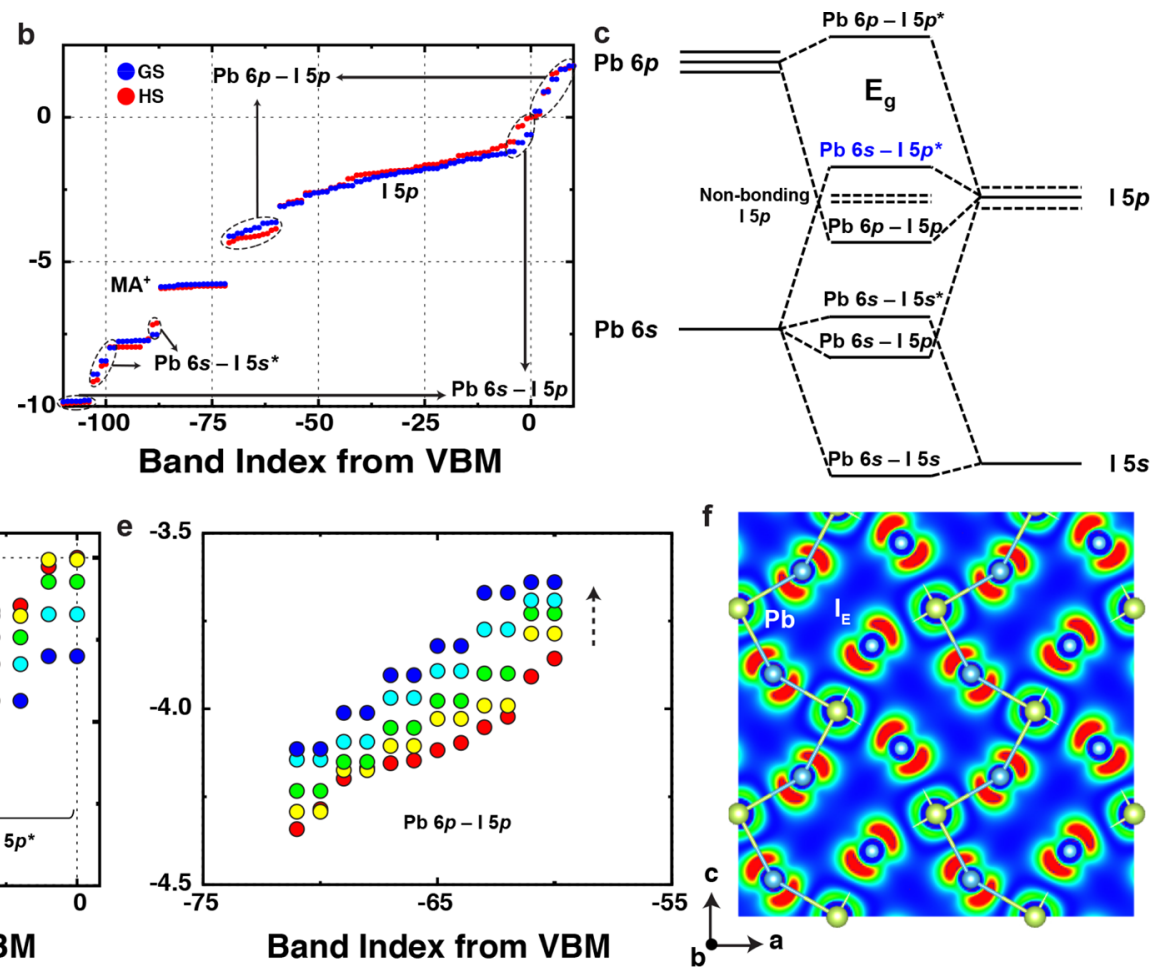

Figure 2. Effect of octahedral tilting on the electronic structure of o-APbI ${ }_{3}$. (a) The computed band diagrams of the GS and HS structures showing high symmetry points on the first Brillouin zone of the orthorhombic structure. The $k$-point-dependent energies of the GS and HS structures are denoted by the blue and red-dotted lines, respectively. (b) The energy eigenstate diagram at the $\Gamma[k=(0,0,0)]$ point. The first band at the $\Gamma$ point, i.e., the valence band maximum (VBM), is indexed as zero. (c) Molecular orbital diagram for the interaction between $\mathrm{Pb}$ and I atoms. (d) The topmost 16 eigenstates below the VBM at the $\Gamma$ point for five increasing amounts $(0$ to 1$)$ of octahedral tilting. (e) The $\mathrm{Pb} 6 \mathrm{p}-\mathrm{I} 5 \mathrm{p}$ bonding states at the $\Gamma$ point for five increasing amounts $(0$ to 1$)$ of octahedral tilting. (f) Electronic charge density contours of the top-most four bands below the VBM at the $\Gamma$ point of the GS structure. The color saturation levels are between 0 (blue) and 0.017 (red) e/ $\mathrm{A}^{3}$. The data shown is for the case of o$\mathrm{MAPbI}_{3}$ for illustration.

structural relaxations were performed with a Gaussian broadening of $0.05 \mathrm{eV}^{41}$ The ions were relaxed until the forces on them were less than $0.01 \mathrm{eV} \AA^{-1}$. The fractional coordinates of all of the ground state structures are given in Tables S4-S23. All schematic representations of the crystal structures were generated using the VESTA program. ${ }^{42}$ For each system, we compute the energy difference, $E_{\mathrm{HS}-\mathrm{GS}}$, between the fully relaxed ground state (GS) orthorhombic structure, and a "high symmetry" (HS) phase in which (i) the cell is fixed to the GS, (ii) the octahedra are fixed and untilted, and (iii) the A-cations are relaxed. In the case of the inorganic perovskites, the HS phase simply corresponds to the high temperature cubic phase (but with the GS lattice parameters). For the hybrid perovskites, it is a hypothetical phase whereby we relax the local MA coordinates but keep them in the same conformation as the orthorhombic phase (see Figure 1). In order to be self-consistent we determined the tolerance factor directly from our DFT electron density, by defining the ionic radii by the volume of a sphere which contains $95 \%$ of the electron density, as done by Filip et al. ${ }^{13}$ For the $\mathrm{MA}^{+}$ion we used both a sphere and a cylinder to determine two effective radii (see SI Figure S1). The two different shapes only changed the effective radius by $0.04 \AA$.

\section{RESULTS}

Effect of Chemical Substitution on Tilting. In order to try and resolve the factors influencing octahedral tilting in $\mathrm{MAPbI}_{3}$, we perform first-principles calculations, based on density functional theory (see Methods), on a variety of inorganic and hybrid perovskite chemistries. We start by considering inorganic $\mathrm{Pb}$-free $\mathrm{ABI}_{3}(\mathrm{~A}=\mathrm{K}, \mathrm{Rb}, \mathrm{Cs}, \mathrm{Fr}$ and $\mathrm{B}=$ $\mathrm{Ca}, \mathrm{Sr}, \mathrm{Ba})$ and subsequently move on to $\mathrm{APbI}_{3}, \mathrm{MABI}_{3}$, and finally $\mathrm{MAPbI}_{3}$. By doing so we can systematically study and discern the role of steric effects (i.e., cation sizes), $\mathrm{Pb}$ electronic effects (e.g., lone pairs), and H-bonding from the MA cation. For each system, we compute the energy difference, $E_{\mathrm{HS}-\mathrm{GS}}$, between the fully relaxed ground state orthorhombic structure (GS), and a "high symmetry" phase which has the octahedra fixed in their untilted positions (HS) (see Methods for details). $E_{\mathrm{HS}-\mathrm{GS}}$ is then a quantitative measure of the propensity of the system to tilt.

We find that, in all the systems studied, the ground state structure is distorted with the orthorhombic tilt pattern $\left(a^{-} b^{+} a^{-}\right)$, i.e., $E_{H S-G S}$ is a nonzero positive value. This might be expected given that all of the materials have a tolerance factor less than 1 , which is the criterion for tilting in perovskites. The calculated lattice parameters are given in SI Table S1, the effective ionic radii in Table S2, and the tolerance factors in Table S3. Figure 1c shows $E_{\mathrm{HS}-\mathrm{GS}}$ as a function of the tolerance factor for each of the cases described above. Considering first the inorganic perovskites without $\mathrm{Pb}$ (red circles in Figure 1c), the tolerance factor appears to be a good descriptor of the propensity for octahedral tilting. The calculations show that as the tolerance factor becomes smaller, i.e. as the cation sizes become less ideally matched to the untilted perovskite cell, the energy gained by tilting becomes systematically greater, which is as expected from classical steric arguments. $^{18}$ It is, however, surprising that a physically oversimplified model appears to give an accurate quantitative description as seen by the precision of the trend line. Interestingly when MA substitutes on the A site (blue squares in Figure 1c), octahedral tilting is strongly stabilized. On the 
a

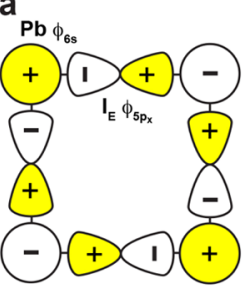

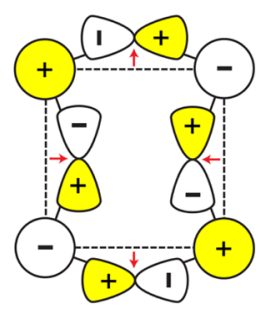

$\mathrm{Pb} 6 \mathrm{~s}-\mathrm{I} 5 p^{*}$ anti-bonding

b

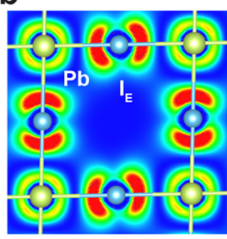

HS

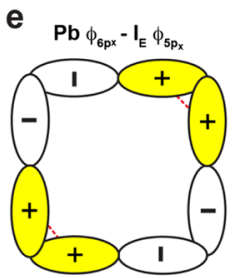

$\mathrm{Pb} 6 p-15 p$ bonding

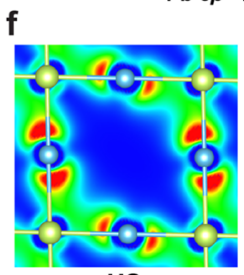

HS
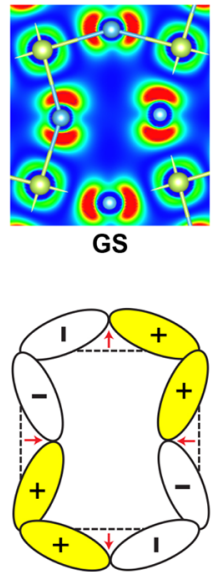

GS

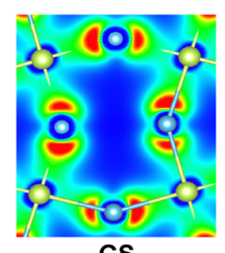

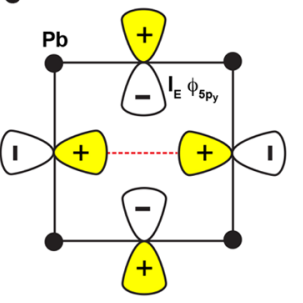

Non-bonding I $5 p$

d
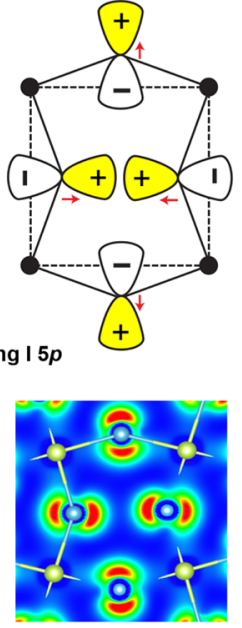

GS
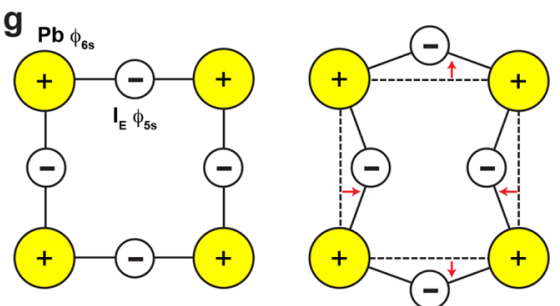

$\mathrm{Pb} 6 \mathrm{~s}-15 \mathrm{~s}^{*}$ anti-bonding

h

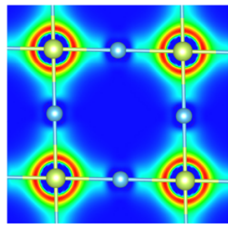

HS

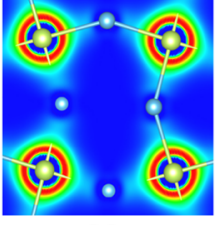

GS

Figure 3. Stabilized and destabilized molecular orbitals through octahedral tilting in o- $\mathrm{APbI}_{3}$. Orbital interaction diagrams illustrating antibonding, nonbonding, and bonding molecular orbitals that aid (top two panels) and hinder (bottom two panels) the octahedral tilting in o-APbI ${ }_{3}$ Both the HS and GS structures are shown. The dotted red lines indicate favorable orbital interactions, and the arrows indicate the direction of the orbital displacement. (a) $\mathrm{Pb} 6 \mathrm{~s}-\mathrm{I} 5 \mathrm{p}^{*}$ antibonding state and (b) its electronic charge density contour corresponding to the four bands immediately below the VBM at the $\Gamma$ point. (c) I 5p nonbonding state and (d) its electronic charge density contour corresponding to bands 9-12 below the VBM at the $\Gamma$ point. (e) $\mathrm{Pb} 6 \mathrm{p}-\mathrm{I} 5 \mathrm{p}$ bonding state and (f) its electronic charge density contour corresponding to the four bands immediately below the first $\mathrm{Pb}$ $6 \mathrm{p}-\mathrm{I} 5 \mathrm{p}$ bonding state (see Figure $2 \mathrm{~b}$ ). (g) $\mathrm{Pb} 6 \mathrm{~s}-\mathrm{I} 5 \mathrm{~s}^{*}$ antibonding state and $(\mathrm{h}$ ) its electronic charge density contour corresponding to bands $100-103$ below the VBM at the $\Gamma$ point. The color saturation levels are between 0 (blue) and $0.017 \mathrm{e} / \mathrm{A}^{3}$ (red). The data shown is for the case of o$\mathrm{MAPbI}_{3}$ for illustration.

other hand, when $\mathrm{Pb}$ is substituted on the $\mathrm{B}$ site, the propensity for octahedral tilting systematically decreases across both series $\left(\mathrm{ABI}_{3}\right.$ and $\left.\mathrm{MABI}_{3}\right)$. Remarkably in the case of $\mathrm{MAPbI}_{3}$ (yellow square in Figure 1c), a hypothetical $\mathrm{APbI}_{3}$ perovskite with the same size A cation as MA (green curve at a tolerance factor of 0.83) would hardly want to tilt at all. In other words the octahedral tilting in $\mathrm{MAPbI}_{3}$ appears to be induced by a mechanism related to the chemical nature of the MA ion, and not its size. The origin of these effects are explored below.

Orbital Interactions. An alternative quantum mechanical way of viewing the classical steric mechanism for octahedral tilting is the second order Jahn-Teller effect whereby the tilts stabilize certain orbital hybridizations. ${ }^{21,23,43-45}$ This could explain the difference in Figure 1c between perovskites with and without $\mathrm{Pb}$; by introducing $\mathrm{Pb}$, new $\mathrm{Pb}$ states such as the lone pair orbitals can rescale (add an extra contribution) to this inherently electronic effect. If this suggestion is correct, it would appear from Figure 1c that overall the octahedral tilts have a destabilizing effect on Pb-based hybridizations. From the analysis below, we demonstrate that tilting in inorganic perovskites primarily results from a stabilizing effect on nonbonding I $5 \mathrm{p}$ orbitals, but that destabilizing contributions from $\mathrm{Pb} 6 \mathrm{p}-\mathrm{I} 5 \mathrm{p}$ bonding states reduces the propensity to tilt in $\mathrm{Pb}$-based perovskites.

To achieve further insight into the origin of tilting, band structure calculations and electron orbital analyses have been performed on o- $\mathrm{APbI}_{3}$. Data for $\mathrm{MAPbI}_{3}$ is displayed here only for illustration of lead-based halide perovskites, and similar qualitative results are found in other $\mathrm{APbI}_{3}$ (see $\mathrm{SI}$ ). As shown in Figure 2a, the computed band structure of o- $\mathrm{APbI}_{3}$ reveals that while the HS structure has only a small band gap (0.03 $\mathrm{eV}$ ), octahedral tilting in the GS structure opens a band gap of about $0.81 \mathrm{eV}$ (see SI Figure S3 for the other chemistries). While these band gaps are most likely underestimated using DFT, it is the trend in behavior that is important here. The electronic structures of the GS and HS configurations were aligned by adjusting their eigenvalues with respect to the vacuum level. Our DFT calculations show that the states 

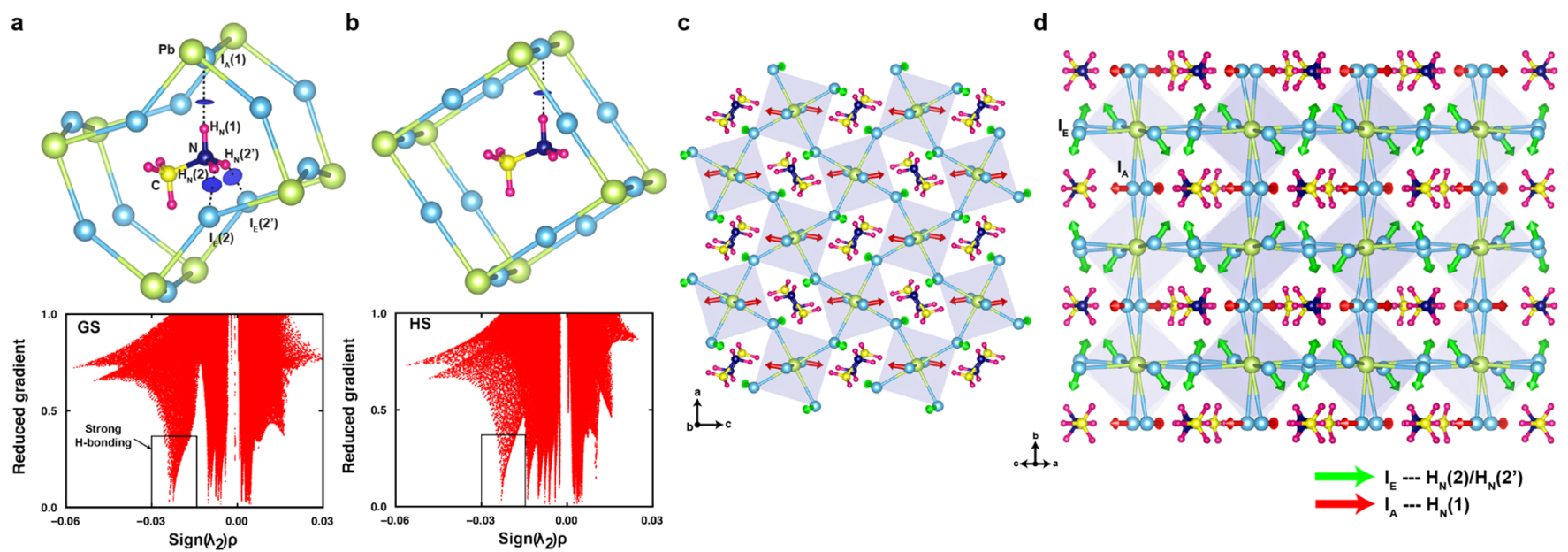

Figure 4. Hydrogen-bonding induced octahedral tilting. (a) Noncovalent interaction (NCI) density isosurface (top) and reduced density gradient $(s)$ as a function of $\operatorname{Sign}\left(\lambda_{2}\right) \rho$ (bottom) for the GS structure. (b) NCI density isosurface (top) and $s$ as a function of $\operatorname{Sign}\left(\lambda_{2}\right) \rho$ (bottom) for the HS structure. $\rho$ is the electron density, and $\lambda_{2}$ is the second eigenvalue of the electron density Hessian matrix. The isosurfaces shown in parts a and $b$ were generated for $s=0.3$ au and -0.03 au $<\rho<-0.015$ au. (c) Hydrogen bonds (indicated by red arrows) between $\mathrm{I}_{\mathrm{A}}$ and $\mathrm{H}_{\mathrm{N}}(1)$ atoms in the GS structure which are directly related to the antiphase rotational mode $\left(a^{-} b^{0} a^{-}\right.$). (d) Hydrogen bonds (indicated by green arrows) between $I_{E}$ and $\mathrm{H}_{\mathrm{N}}(2) / \mathrm{H}_{\mathrm{N}}\left(2^{\prime}\right)$ atoms in the GS structure which are correlated with both in-phase and antiphase rotational modes, simultaneously $\left(\mathrm{a}^{-} \mathrm{b}^{+} \mathrm{a}^{-}\right)$.

toward the top of the valence band region are stabilized by the octahedral tilting, and this agrees with previous studies on the correlation between tilting and band gap opening. ${ }^{28}$ The character of the wave functions at the $\Gamma$-point $[k=(0,0,0)]$ has been analyzed to identify the orbital interaction relevant to octahedral tilting in the GS structure. To do this, the band index is labeled in descending order of energy beginning at the valence band maximum (VBM), which is given a band index of 0 . As shown in Figure $2 b$, the top of the valence band consists of $\mathrm{Pb} 6 \mathrm{~s}-\mathrm{I} 5 \mathrm{p}^{*}$ antibonding states (whose electronic density profile is shown in Figure 2f), followed by (i) nonbonding I $5 p$ states, (ii) $\mathrm{Pb} 6 \mathrm{p}-\mathrm{I} 5 \mathrm{p}$ bonding states, (iii) $\mathrm{MA}^{+}$(or more generally $\mathrm{A}^{+}$) states, (iv) $\mathrm{Pb} 6 \mathrm{~s}-\mathrm{I} 5 \mathrm{~s}^{*}$ antibonding states, and (v) $\mathrm{Pb} 6 \mathrm{~s}-\mathrm{I} 5 \mathrm{p}$ bonding states. The partial charge densities of these states are shown in SI Figure S4. On the basis of these results, a $\mathrm{Pb}-\mathrm{I}$ molecular orbital diagram is constructed and shown in Figure 2c.

According to the energy band index diagram shown in Figure $2 \mathrm{~b}$, the only molecular orbital states that are stabilized by the octahedral tilting are the $\mathrm{Pb} 6 \mathrm{~s}-\mathrm{I} 5 \mathrm{p} *$ antibonding and nonbonding I $5 \mathrm{p}$ orbitals. This is consistent with our calculated partial densities of states (PDOS) results (see SI Figure S5). In Figure $2 \mathrm{~d}$ the Kohn-Sham $(\mathrm{K}-\mathrm{S})$ energies of the $\mathrm{Pb} 6 \mathrm{~s}-\mathrm{I} 5 \mathrm{p}$ * antibonding and nonbonding I $5 \mathrm{p}$ states are shown as a function of the amount of octahedral tilting, and found to all decrease with increasing tilt angle. The points labeled 0 to 1 represent the degree of tilting between the HS and GS structures, which correspond to the five points between the HS and GS in Figure 1c (inset). The decrease in the $\mathrm{K}-\mathrm{S}$ energy with increasing amount of octahedral tilting can be attributed to the stabilization of the $\mathrm{Pb} 6 \mathrm{~s}-\mathrm{I} 5 \mathrm{p}^{*}$ antibonding and nonbonding I $5 p$ states near the VBM. By contrast, the $\mathrm{Pb}$ $6 \mathrm{p}-\mathrm{I} 5 \mathrm{p}$ bonding states at higher band indices are destabilized by the octahedral tilting as shown in Figure $2 \mathrm{e}$.

To understand the structural stabilization of $\mathrm{o}-\mathrm{APbI}_{3}$ in terms of the electronic structure of the $\mathrm{Pb} 6 \mathrm{~s}-\mathrm{I} 5 \mathrm{p} *$ antibonding and nonbonding I 5p molecular orbitals, we propose two orbital interaction mechanisms which are schematically depicted in Figure 3. As shown in Figure 3a, I $5 \mathrm{p}_{x}\left(\phi_{5 \mathrm{p}_{x}}\right)$ orbitals tend to displace from the linear $\mathrm{Pb}-\mathrm{I}_{\mathrm{E}}-\mathrm{Pb}$ bond line to stabilize the orbital interaction energy in the $\mathrm{Pb} 6 \mathrm{~s}-\mathrm{I} 5 \mathrm{p}^{*}$ antibonding molecular orbitals, which is in accordance with the computed electronic density profile of the $\mathrm{Pb} 6 \mathrm{~s}-\mathrm{I} 5 \mathrm{p} *$ antibonding states presented in Figure $3 \mathrm{~b}$. Furthermore, the nonbonding I $5 \mathrm{p}_{y}$ orbitals $\left(\phi_{5 \mathrm{p}_{y}}\right)$ are also displaced away from the linear $\mathrm{Pb}-\mathrm{I}_{\mathrm{E}}-$ $\mathrm{Pb}$ bond line (see Figure $3 \mathrm{c}$ ). The computed electronic density profile of the nonbonding I $5 p$ orbitals support this orbital interaction as shown in Figure 3d. All these computed results thus clearly indicate that the octahedral tilting in $\mathrm{o}-\mathrm{APbI}_{3}$ is closely linked with the stabilization of the $\mathrm{Pb} 6 \mathrm{~s}-\mathrm{I} 5 \mathrm{p}^{*}$ antibonding and nonbonding I $5 p$ molecular orbitals. Equivalent orbital interaction mechanisms for the $\mathrm{Pb} 6 \mathrm{p}-\mathrm{I} 5 \mathrm{p}$ bonding and $\mathrm{Pb} 6 \mathrm{~s}-\mathrm{I} 5 \mathrm{~s}^{*}$ antibonding states have been sketched in Figure $3 \mathrm{e}-\mathrm{h}$, which we argue plays a role in destabilizing octahedral tilts. It is the sum of all of these contributions which determines the propensity of the octahedra to tilt.

Hydrogen Bonding. So far we have attempted to rationalize octahedral tilting in inorganic perovskites on the basis of tilting induced orbital hybridizations, otherwise known as the second order Jahn-Teller effect. This has successfully accounted for the behavior of $E_{\mathrm{HS}-\mathrm{GS}}$ as a function of tolerance factor, and the effect of $\mathrm{Pb}$ substituted perovskites (Figure 1c). The greater propensity for hybrid perovskites to tilt compared to inorganic perovskites with similar ionic sizes still needs understanding. This cannot be explained as a second order Jahn-Teller effect since the MA electronic states are either seen to be unaltered by tilting, or slightly destabilized (see Figure 2b).

It has been shown previously that strong hydrogen bonding exists between the MA cation and the iodine framework, and this affects octahedral tilting. ${ }^{31}$ We begin by re-examining this interplay between hydrogen bonding and tilting. In a previous study it was found that hydrogen bonding is noticeably weaker in the HS structure. ${ }^{46}$ This was demonstrated by calculating the noncovalent interaction $(\mathrm{NCI})^{47}$ contours of both structures, and these are shown in Figure 4a,b. For the GS structure (Figure 4a), the NCI contour shows the presence of three 
strong hydrogen bonding interactions: (i) between $\mathrm{H}_{\mathrm{N}}(1)$ and the axial iodine atom labeled $\mathrm{I}_{\mathrm{A}}(1)$, (ii) between $\mathrm{H}_{\mathrm{N}}(2)$ and the equatorial iodine atom labeled $\mathrm{I}_{\mathrm{E}}(2)$, and (iii) between $\mathrm{H}_{\mathrm{N}}\left(2^{\prime}\right)$ and $\mathrm{I}_{\mathrm{E}}\left(2^{\prime}\right)$. The three contours clearly indicate the presence of three strong hydrogen bonds and two types of bonding modes (axial and equatorial). However, in the HS structure (Figure $4 \mathrm{~b})$ there is only one contour which is between $\mathrm{H}_{\mathrm{N}}(1)$ and $I_{A}(1)$, indicating that the $H_{N}(1)-I_{A}(1)$ interaction is slightly weakened in the $\mathrm{HS}$ structure and that the other $\mathrm{H}-\mathrm{I}$ interactions are absent. The NCI contours are supported by plots of the reduced density gradient $(s)$ as a function of $\operatorname{Sign}\left(\lambda_{2}\right) \rho$ where $\rho$ is the electron density and $\lambda_{2}$ is the second eigenvalue of the electron density Hessian matrix. A trough and singularity in the reduced density gradient indicate the presence of a noncovalent interaction. $\lambda_{2}$ can be either positive or negative depending on the type of interaction. When there is hydrogen bonding, $\lambda_{2}<0$. On the other hand, $\lambda_{2}>0$ indicates there are nonbonded interactions such as steric repulsion. In the case of van der Waals interactions, $\lambda_{2}$ approaches zero. $\rho$ indicates the interaction strength, and thus higher values of $|\rho|$ suggest stronger interactions. The isosurfaces shown in Figure $4 \mathrm{a}, \mathrm{b}$ were generated for $s=0.3 \mathrm{au}$ and $-0.03 \mathrm{au}<\rho<-0.015$ au. Thus, a singularity in the reduced gradient density within this window indicates strong hydrogen bonds. The reduced density gradient plots show that hydrogen bonds are present in both the GS and HS structures but that they are stronger in the GS structure.

In Figure 4c,d we also sketch the pattern of iodine motions expected from the two different hydrogen modes in the GS structure. The bonding between $\mathrm{I}_{\mathrm{A}}$ and $\mathrm{H}_{\mathrm{N}}(1)$ atoms is indicated by red arrows (mode 1 ), and that between $\mathrm{I}_{\mathrm{E}}$ and $\mathrm{H}_{\mathrm{N}}(2) / \mathrm{H}_{\mathrm{N}}\left(2^{\prime}\right)$ atoms is indicated by green arrows (mode 2 ). This pattern of iodine displacements expected from hydrogen bonding corresponds perfectly with the resulting octahedral tilting of the orthorhombic phase. It is interesting to note that while mode 1 induces only the antiphase octahedral rotation, mode 2 correlates with both in-phase and antiphase rotations.

\section{DISCUSSION}

The analysis of the first-principles calculations presented above suggests that the degree of octahedral tilting in hybrid halide perovskites depends on a subtle balance of orbital interactions between the inorganic species (second order Jahn-Teller effects), and hydrogen bonding between the MA cation and the anion framework. Let us consider the case of the prototypical hybrid perovskite $\mathrm{MAPbI}_{3}$. If we could hypothetically remove hydrogen bonding from this system by, for example, replacing MA by an inorganic (or H-bonding free organic) cation of the same size, we would expect $E_{\mathrm{HS}-\mathrm{GS}}$ to be negligible, as shown by the arrow in Figure 1c. This means that octahedral tilting does not favor orbital hybridizations; the combined effects of stabilizing and destabilizing orbital interactions almost exactly cancel. We then conclude, returning to $\mathrm{MAPbI}_{3}$, that without hydrogen bonding this perovskite would remain untilted.

If hydrogen bonding is responsible for the orthorhombic phase, could it also be responsible for the room-temperature tilted tetragonal phase? This problem is beyond the scope of the current paper, especially given the complex nature of the partially disordered MA species within this phase. However, we note that the hydrogen bonds between $\mathrm{I}_{\mathrm{A}}$ and $\mathrm{H}_{\mathrm{N}}(1)$ atoms, which we predict to exist even in the untilted orthorhombic phase (i.e., will induce a force on the atoms), are directly related to the antiphase rotational mode which constitutes the tetragonal structure. We note related discussions in a recent study to which we refer for more in-depth analysis. ${ }^{48}$

Finally, we briefly consider the consequence of hydrogen bonding for photovoltaic applications. In Figure 5, we plot the
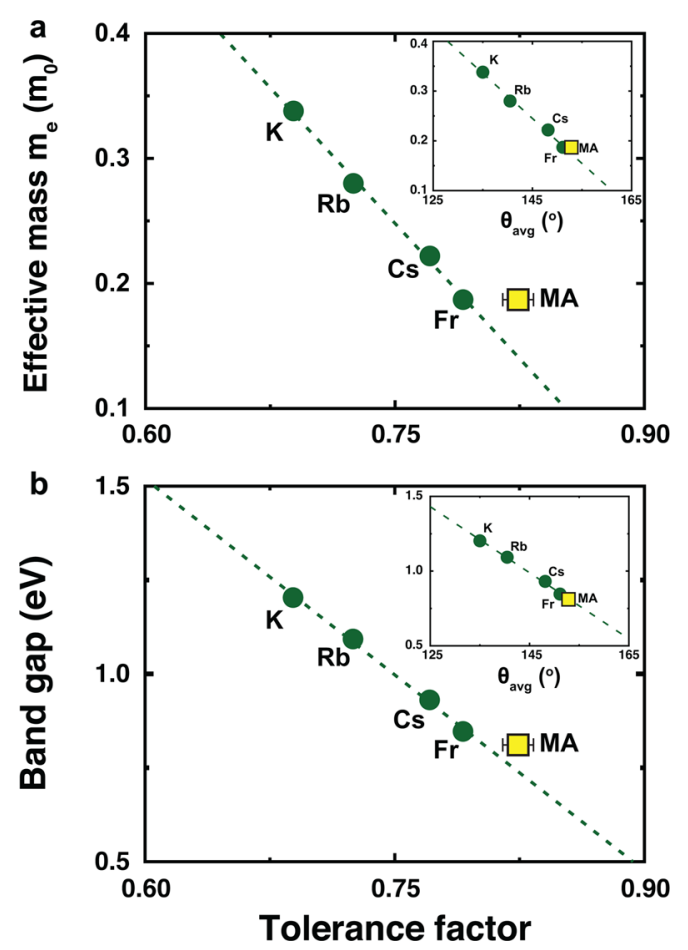

Figure 5. Beyond steric effects for photovoltaic engineering. The computed electron effective mass $\left(m_{\mathrm{e}}\right)(\mathrm{a})$ and band gaps $(\mathrm{b})$ of o$\mathrm{APbI}_{3}$ (circles) and o-MAPbI (square) plotted as a function of the tolerance factor. The insets show how the two quantities vary with average tilt angle.

electron effective mass at the $\Gamma$ point and band gap as a function of tolerance factor. As expected, across the inorganic series, as the tolerance factor reduces the effective electron masses, band gaps increase due to enhanced octahedral tilting. However, $\mathrm{MAPbI}_{3}$ does not fit the trend lines, which would otherwise have predicted $\mathrm{MAPbI}_{3}$ to exhibit a favorably reduced effective electron mass and band gap. Instead, hydrogen bonding has enhanced octahedral tilting beyond that expected from the tolerance factor, which in turn has increased the effective electron mass and band gap (see Figure 5, insets). One can imagine that, in order to design halide perovskites with enhanced carrier mobilities and reduced band gaps, hydrogen bonding should be minimized. Alternatively, one could consider engineering the direction of the hydrogen bonds to disfavor octahedral tilting. This appears to be the case, for example, in $\mathrm{FAPbI}_{3}\left(\mathrm{FA}=\mathrm{NH}_{2} \mathrm{CHNH}_{2}\right)$, which has hydrogen bonds on both sides of the molecule ${ }^{14}$ rendering the system pseudocubic.

\section{CONCLUDING REMARKS}

To conclude, first-principles calculations have been performed on various inorganic and hybrid halide perovskites in an attempt to resolve the physical origin of octahedral tilting in these systems. While we find that orbital interactions dominate in inorganic perovskites, in line with standard arguments, hydrogen bonding plays a critical role in stabilizing octahedral tilts in hybrid perovskites such as $\mathrm{MAPbI}_{3}$. Since octahedral tilts affect band gaps and carrier masses, engineering the strength 
and direction of the hydrogen bonding between the organic molecule and the anion framework could be considered as a future avenue for optimizing solar cell efficiency. Understanding new parameters that control tilting is not only important for photovoltaic engineering in halides, but has implications more generally for all perovskites where tilts influence many functional properties including magnetism, ferroelectricity, and magnetoresistance.

\section{ASSOCIATED CONTENT}

\section{S Supporting Information}

The Supporting Information is available free of charge on the ACS Publications website at DOI: 10.1021/acs.chemmater.6b00968.

Figures S1-S5, illustrating the computed effective radius of the MA cation, and the tolerance factors, band structures, partial charge density contours, and density of states of the various perovskites considered, and Tables S1-S23, giving the computed lattice parameters, effective ionic radii, tolerance factors, tilt angles, energy barriers, and fractional coordinates of the same set of perovskites (PDF)

\section{AUTHOR INFORMATION}

\section{Corresponding Authors}

*E-mail: pdb1000@cam.ac.uk.

*E-mail: akc30@cam.ac.uk.

*E-mail: hmjang@postech.ac.kr.

\section{Author Contributions}

J.-H.L. and N.C.B. contributed equally.

\section{Notes}

The authors declare no competing financial interest.

\section{ACKNOWLEDGMENTS}

We thank Marina Filip at University of Oxford for helpful discussions. The work at POSTECH was supported by National Research Foundation (NRF) Grants funded by the Korea Government (MSIP) (Grants 2012R 1A1A2041628 and 2013R 1A2A2A01068274). At the University of Cambridge, the work was funded by the Winton Programme for the Physics of Sustainability. The work at Imperial College London was supported by the Royal Commission for the Exhibition of 1851 and Imperial College's Junior Research Fellowships. The calculations were performed at the Cambridge HPCS and the UK National Supercomputing Service, ARCHER. Access to the latter was obtained via the UKCP consortium and funded by EPSRC under Grant EP/K014560/1. All necessary computational data is included in the paper or Supporting Information.

\section{REFERENCES}

(1) Kojima, A.; Teshima, K.; Shirai, Y.; Miyasaka, T. Organometal halide perovskites as visible-light sensitizers for photovoltaic cells. J. Am. Chem. Soc. 2009, 131, 6050-6051.

(2) Lee, M. M.; Teuscher, J.; Miyasaka, T.; Murakami, T. N.; Snaith, H. J. Efficient hybrid solar cells based on meso-superstructured organometal halide perovskites. Science 2012, 338, 643-647.

(3) Chung, I.; Lee, B.; He, J.; Chang, R. P.; Kanatzidis, M. G. Allsolid-state dye-sensitized solar cells with high efficiency. Nature 2012, 485, 486-489.

(4) Burschka, J.; Pellet, N.; Moon, S.-J.; Humphry-Baker, R.; Gao, P.; Nazeeruddin, M. K.; Grätzel, M. Sequential deposition as a route to high-performance perovskite-sensitized solar cells. Nature 2013, 499, 316-319.
(5) Liu, M.; Johnston, M. B.; Snaith, H. J. Efficient planar heterojunction perovskite solar cells by vapour deposition. Nature 2013, 501, 395-398.

(6) Gao, P.; Grätzel, M.; Nazeeruddin, M. K. Organohalide lead perovskites for photovoltaic applications. Energy Environ. Sci. 2014, 7, $2448-2463$.

(7) Green, M. A.; Ho-Baillie, A.; Snaith, H. J. The emergence of perovskite solar cells. Nat. Photonics 2014, 8, 506-514.

(8) Grätzel, M. The light and shade of perovskite solar cells. Nat. Mater. 2014, 13, 838-842.

(9) McGehee, M. D. Perovskite solar cells: continuing to soar. Nat. Mater. 2014, 13, 845-846.

(10) Hoke, E. T.; Slotcavage, D. J.; Dohner, E. R.; Bowring, A. R.; Karunadasa, H. I.; McGehee, M. D. Reversible photo-induced trap formation in mixed-halide hybrid perovskites for photovoltaics. Chem. Sci. 2015, 6, 613-617.

(11) Jeon, N. J.; Noh, J. H.; Kim, Y. C.; Yang, W. S.; Ryu, S.; Seok, S. I. Solvent engineering for high-performance inorganic-organic hybrid perovskite solar cells. Nat. Mater. 2014, 13, 897.

(12) NREL. Research Cell Efficiency Records; 2015; http://www.nrel. gov/ncpv/images/efficiency_chart.jpg.

(13) Filip, M. R.; Eperon, G. E.; Snaith, H. J.; Giustino, F. Steric engineering of metal-halide perovskites with tunable optical band gaps. Nat. Commun. 2014, 5, 5757.

(14) Amat, A.; Mosconi, E.; Ronca, E.; Quarti, C.; Umari, P.; Nazeeruddin, M. K.; Grätzel, M.; De Angelis, F. Cation-induced bandgap tuning in organohalide perovskites: Interplay of spin-orbit coupling and octahedra tilting. Nano Lett. 2014, 14, 3608-3616.

(15) Baikie, T.; Fang, Y.; Kadro, J. M.; Schreyer, M.; Wei, F.; Mhaisalkar, S. G.; Graetzel, M.; White, T. J. Synthesis and crystal chemistry of the hybrid perovskite $\left(\mathrm{CH}_{3} \mathrm{NH}_{3}\right) \mathrm{PbI}_{3}$ for solid-state sensitised solar cell applications. J. Mater. Chem. A 2013, 1, 56285641.

(16) Thomas, N. W. The compositional dependence of octahedral tilting in orthorhombic and tetragonal perovskites. Acta Crystallogr., Sect. B: Struct. Sci. 1996, 52, 16-31.

(17) Woodward, P. M. Octahedral tilting in perovskites. I. Geometrical considerations. Acta Crystallogr., Sect. B: Struct. Sci. 1997, 53, 32-43.

(18) Woodward, P. M. Octahedral tilting in perovskites. II. Structure stabilizing forces. Acta Crystallogr., Sect. B: Struct. Sci. 1997, 53, 44-66.

(19) Howard, C. J.; Stokes, H. Group-theoretical analysis of octahedral tilting in perovskites. Acta Crystallogr., Sect. B: Struct. Sci. 1998, 54, 782-789.

(20) Stokes, H. T.; Kisi, E. H.; Hatch, D. M.; Howard, C. J. Grouptheoretical analysis of octahedral tilting in ferroelectric perovskites. Acta Crystallogr., Sect. B: Struct. Sci. 2002, 58, 934-938.

(21) Lufaso, M. W.; Woodward, P. M. Jahn-Teller distortions, cation ordering and octahedral tilting in perovskites. Acta Crystallogr., Sect. B: Struct. Sci. 2004, 60, 10-20.

(22) Angel, R.; Zhao, J.; Ross, N. General rules for predicting phase transitions in perovskites due to octahedral tilting. Phys. Rev. Lett. 2005, 95, 025503.

(23) Carpenter, M. A.; Howard, C. J. Symmetry rules and strain/ order-parameter relationships for coupling between octahedral tilting and cooperative Jahn-Teller transitions in $\mathrm{ABX}_{3}$ perovskites. I. Theory. Acta Crystallogr., Sect. B: Struct. Sci. 2009, 65, 134-146.

(24) Glazer, A. Classification of tilted octahedra in perovskites. Acta Crystallogr., Sect. B: Struct. Crystallogr. Cryst. Chem. 1972, 28, 33843392.

(25) Goldschmidt, V. The laws of crystal chemistry. Naturwissenschaften 1926, 14, 477-85.

(26) Kieslich, G.; Sun, S.; Cheetham, A. K. Solid-state principles applied to organic-inorganic perovskites: new tricks for an old dog. Chem. Sci. 2014, 5, 4712-4715.

(27) Kieslich, G.; Sun, S.; Cheetham, A. K. An extended Tolerance Factor approach for organic-inorganic perovskites. Chem. Sci. 2015, 6, $3430-3433$. 
(28) Kim, J.; Lee, S.-C.; Lee, S.-H.; Hong, K.-H. Importance of Orbital Interactions in Determining Electronic Band Structures of Organo-Lead Iodide. J. Phys. Chem. C 2015, 119, 4627-4634.

(29) Motta, C.; El-Mellouhi, F.; Kais, S.; Tabet, N.; Alharbi, F.; Sanvito, S. Revealing the role of organic cations in hybrid halide perovskite $\mathrm{CH}_{3} \mathrm{NH}_{3} \mathrm{PbI}_{3}$. Nat. Commun. 2015, 6, 7026 .

(30) Weller, M. T.; Weber, O. J.; Henry, P. F.; Di Pumpo, A. M.; Hansen, T. C. Complete structure and cation orientation in the perovskite photovoltaic methylammonium lead iodide between 100 and 352 K. Chem. Commun. (Cambridge, U. K.) 2015, 51, 4180-4183.

(31) Lee, J.-H.; Bristowe, N. C.; Bristowe, P. D.; Cheetham, A. K. Role of hydrogen-bonding and its interplay with octahedral tilting in $\mathrm{CH}_{3} \mathrm{NH}_{3} \mathrm{PbI}_{3}$. Chem. Commun. (Cambridge, U. K.) 2015, 51, 64346437.

(32) Blöchl, P. E. Projector augmented-wave method. Phys. Rev. B: Condens. Matter Mater. Phys. 1994, 50, 17953.

(33) Kresse, G.; Joubert, D. From ultrasoft pseudopotentials to the projector augmented-wave method. Phys. Rev. B: Condens. Matter Mater. Phys. 1999, 59, 1758.

(34) Kresse, G.; Hafner, J. Ab initio molecular dynamics for liquid metals. Phys. Rev. B: Condens. Matter Mater. Phys. 1993, 47, 558.

(35) Kresse, G.; Furthmüller, J. Efficient iterative schemes for $a b$ initio total-energy calculations using a plane-wave basis set. Phys. Rev. B: Condens. Matter Mater. Phys. 1996, 54, 11169.

(36) Kresse, G.; Furthmüller, J. Efficiency of ab-initio total energy calculations for metals and semiconductors using a plane-wave basis set. Comput. Mater. Sci. 1996, 6, 15-50.

(37) Kresse, G.; Hafner, J. Ab initio molecular-dynamics simulation of the liquid-metal-amorphous-semiconductor transition in germanium. Phys. Rev. B: Condens. Matter Mater. Phys. 1994, 49, 14251.

(38) Thonhauser, T.; Cooper, V. R.; Li, S.; Puzder, A.; Hyldgaard, P.; Langreth, D. C. Van der Waals density functional: Self-consistent potential and the nature of the van der Waals bond. Phys. Rev. B: Condens. Matter Mater. Phys. 2007, 76, 125112.

(39) Klimeš, J.; Bowler, D. R.; Michaelides, A. Van der Waals density functionals applied to solids. Phys. Rev. B: Condens. Matter Mater. Phys. 2011, 83, 195131.

(40) Blöchl, P. E.; Jepsen, O.; Andersen, O. K. Improved tetrahedron method for Brillouin-zone integrations. Phys. Rev. B: Condens. Matter Mater. Phys. 1994, 49, 16223.

(41) Elsässer, C.; Fähnle, M.; Chan, C.; Ho, K. Density-functional energies and forces with Gaussian-broadened fractional occupations. Phys. Rev. B: Condens. Matter Mater. Phys. 1994, 49, 13975.

(42) Momma, K.; Izumi, F. VESTA: a three-dimensional visualization system for electronic and structural analysis. J. Appl. Crystallogr. 2008, $41,653-658$.

(43) Kristoffel, N.; Konsin, P. Pseudo-Jab-Teller Effect and Second Order Phase Transitions in Crystals. Phys. Status Solidi B 1967, 21, K39-K43.

(44) Pearson, R. G. The second-order Jahn-Teller effect. J. Mol. Struct.: THEOCHEM 1983, 103, 25-34.

(45) Garcia-Fernandez, P.; Aramburu, J. a.; Barriuso, M. T.; Moreno, $\mathrm{M}$. Key role of covalent bonding in octahedral tilting in perovskites. J. Phys. Chem. Lett. 2010, 1, 647-651.

(46) These contours were not illustrated correctly in ref 30, but this did affect the conclusions.

(47) Contreras-García, J.; Johnson, E. R.; Keinan, S.; Chaudret, R.; Piquemal, J.-P.; Beratan, D. N.; Yang, W. NCIPLOT: a program for plotting noncovalent interaction regions. J. Chem. Theory Comput. 2011, 7, 625-632.

(48) Lee, J. H.; Lee, J.-H.; Kong, E.-H.; Jang, H. M. The nature of hydrogen-bonding interaction in the prototypic hybrid halide perovskite, tetragonal $\mathrm{CH}_{3} \mathrm{NH}_{3} \mathrm{PbI}_{3}$. Sci. Rep. 2016, 6, 21687. 